\title{
REVIEW
}

\section{Primary care referral protocol for carpal tunnel syndrome}

\author{
A C Hayward, M J Bradley, F D Burke
}

Postgrad Med J 2002;78:149-152

Carpal tunnel syndrome is an extremely common upper limb nerve compression syndrome, widely distributed in the community. There are a variety of treatment options which may be applied to the syndrome, depending on the severity of symptoms. Some options are available in a primary care setting, others require secondary referral. This paper is a detailed review of the available literature and provides a protocol that could be used to assist in the referral of patients from primary care.

\section{INCIDENCE OF CARPAL TUNNEL SYNDROME}

The available information in the literature on carpal tunnel syndrome (CTS) falls into three categories: operative rates from secondary treatment centres per 100000 population per year; assessment of incidence based on data from secondary care, in general; and prevalence estimation derived from community studies.

The Derby Hand Centre found an overall operative incidence of 61 per 100000 population per year in 1989. ${ }^{1}$ Other studies have shown a higher incidence. Keller and co-workers identified an overall incidence of 144 decompressions per 100000 population per year in America in
1993. ${ }^{2}$ The average was derived from 21 centres in the State of Maine. The variation between the participating centres was remarkably wide (from 82 to 287 per 100000 population), indicating a more than threefold variation for which no obvious cause could be identified. Such large variations in surgery rates probably reflect differences in patient and general practitioner awareness, coupled with varying thresholds for operative treatment, rather than a spectrum of prevalence of the disease. The average decompression rate for the 11 centres in the Trent region of the UK in 1996 is less than half the average found in Maine (56 decompressions per 100000 population per year). The variation between the 11 centres was also lower (43-47 per 100000 population per year).

Nordstrum and co-workers reported that the incidence of CTS in America was 346 per 100000 population per year. ${ }^{3}$ The investigators used a community medical database in Wisconsin to find all patients who were receiving medical or surgical treatment for CTS.

The prevalence of CTS in a Dutch community has been investigated. ${ }^{4} \mathrm{~A}$ random sample of

Abbreviations: CTS, carpal tunnel syndrome; NSAIDs, non-steroidal anti-inflammatory agents

\section{Box 1: Key points to advise primary care workers about CTS}

A thorough literature review of CTS management has identified the following findings. These findings have been used to develop referral guidelines (fig 1):

- CTS is often a progressive condition, however many patients have a satisfactory response to work modification or conservative management.

- If CTS does not respond to conservative management within six months, it is unlikely to respond at all.

- Steroid injection provides effective temporary relief, but symptoms are likely to return within a few months. For this reason, it is only recommended for pregnant patients, because CTS is likely to resolve after pregnancy.

- Steroid injection can lead to complications such as injection of the median nerve and should only be performed by those with adequate training.

- Patients who are unlikely to respond to conservative management include those who have constant symptoms for more than six months at presentation and those who have already failed to respond to a trial of conservative management. These patients should be referred in order to be considered for surgery.

- Patients who are aged over 50 are less likely to respond to conservative management than younger patients. However, if the disease is not severe (Phalen's test is negative or the test takes longer than 30 seconds to become positive), then they may respond to a trial of conservative treatment.

See end of article for authors' affiliations

Correspondence to: Professor F D Burke, Pulvertaft Hand Centre, Derbyshire Royal Infirmary, London Road, Derby DEI 2QY, UK

Submitted 2 July 2001 Accepted 18 September 200
- Operative treatment leads to resolution of symptoms in over $80 \%$ of patients, but outcome tends to be less satisfactory in those with severe disease or those who have had symptoms for over three years.

- Scar tenderness can be a significant problem for some patients after their operation.

- Most trials of conservative management have used work modification, splinting of the wrist in a neutral position during the night (and during the day when symptomatic), and the use of oral NSAIDs. The evidence for the use of diuretics, oral steroids, or pyridoxine is unconvincing.

- Work modification may include: an ergonomic assessment of the workstation, avoidance of heavy work, and avoidance of repetitive hand and wrist movements, or if this is not possible, taking regular breaks.

- If a patient is being referred for surgery, they should be aware of the general character of the procedure and be agreeable to having surgery if it is deemed necessary. 


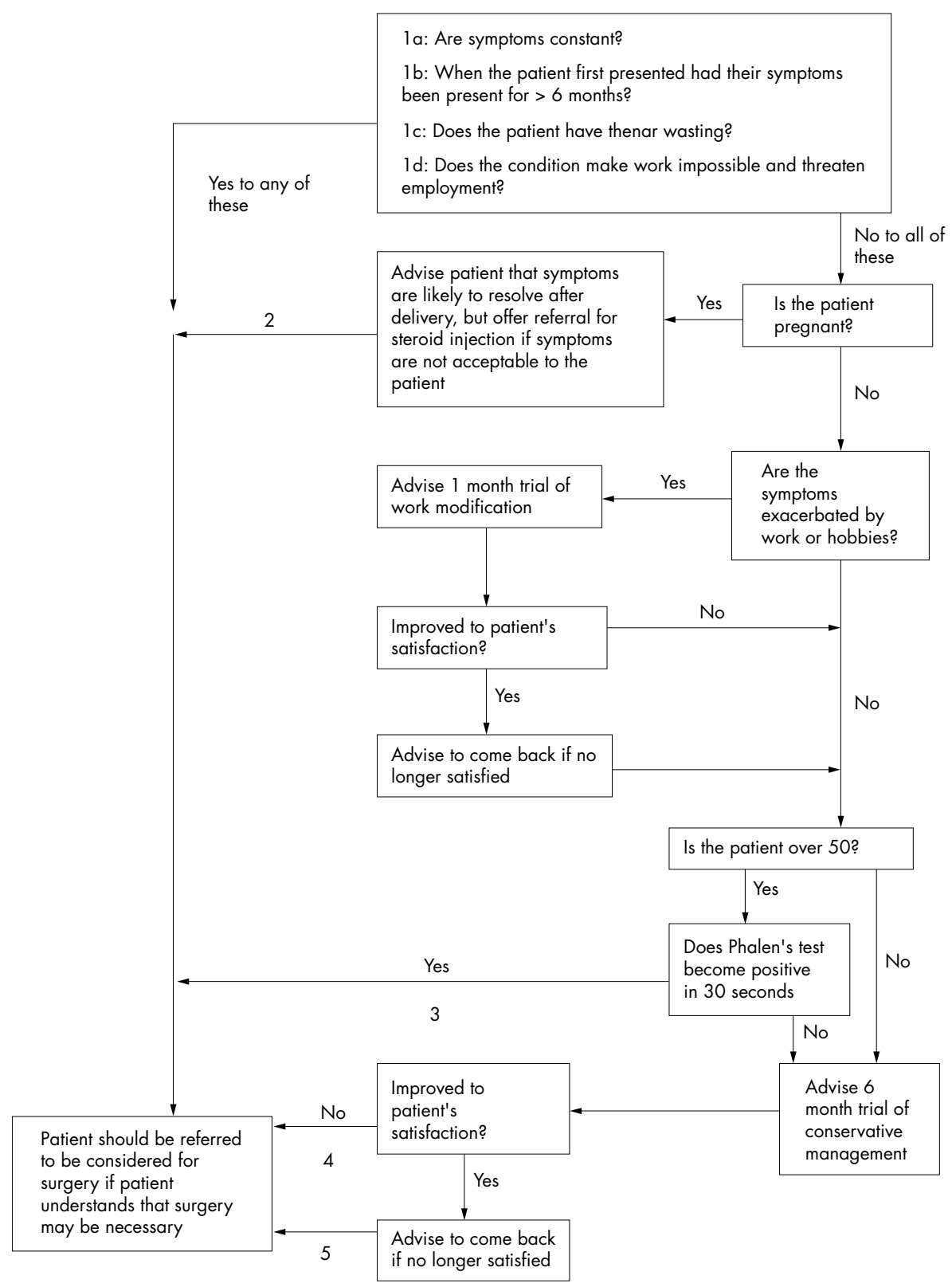

Figure 1 Proposed referral guidelines for carpal tunnel syndrome.

adults was asked specifically about symptoms consistent with the criteria for CTS. The prevalence rate for detected CTS in females was $3.4 \%$ (compared with $0.6 \%$ in males). However, the rate of undetected CTS in females was $5.8 \%$, revealing a high reservoir of undiagnosed cases within the community. The diagnosis in these cases had been confirmed by nerve conduction studies. The figures are a minimum estimate, because 14 out of 45 cases with appropriate symptoms refused to undergo nerve conduction studies and thus were presumed normal. It is not clear from the paper whether the third of patients who were diagnosed were the most severe cases in the study. Ferry et al performed a similar study in the UK by using case histories and nerve conduction assessment. ${ }^{5}$ A prevalence estimate of between $7 \%$ and $16 \%$ was found, depending on the stringency of the neurological assessment (that is, whether motor as well as sensory nerve changes were present). The only difference in results between this study and that of de Krom and co-workers is that the prevalence in males and females was found to be equal.

The evidence in the literature indicates that CTS is very common and that there are many undiagnosed cases. A rational policy for referral from primary care seems prudent for a condition that leads to surgical decompression in almost 3000 cases each year in a 5000000 population. ${ }^{6}$

\section{ASSOCIATED FACTORS}

A positive association has been identified between CTS and obesity, diabetes, pregnancy, rheumatoid arthritis, and hypothyroidism. ${ }^{7}$ The association between CTS and occupation remains controversial. There is an agreed association between CTS and vibration white finger, although the pathophysiology remains uncertain. Such cases may have the classical features of nerve damage arising from compression or, alternatively, they may arise from direct vibration injury to the nerve. Stromberg and co-workers identified histological evidence of vibration damage to the posterior interosseous nerve in patients who have been exposed to significant amounts of vibration in the past and similar features may be present in the median nerve. ${ }^{8}$

Rapid repetitive or strenuous hand activity may exacerbate the symptoms of CTS. The condition is considered by some to be caused by work. This is justified on the basis of forceful or repetitive grasping causing flexor tenosynovitis, thereby 
I have assessed my patient using the Derby Carpal Tunnel Referral Protocol and wish to refer the patient because:

(Please tick the appropriate box/boxes)

la They have constant symptoms

1b At presentation their symptoms had already been present for more than 6 months.

1c They have thenar wasting.

1d Their condition makes it impossible to work and threatens employment.

2 The patient is pregnant-please consider steroid injection.

3 The patient is over 50 years old and Phalen's test becomes positive in less than 30 seconds.

4 The patient has failed to respond to a 6 month trial of conservative management.

5 The patient responded to a 6 month trial of conservative management, but their symptoms have now returned.

6 The patient understands that surgery may be necessary.

Other reasons (please specify)

Figure 2 Referral letter for general practitioners referring patients with carpal tunnel syndrome.

creating increased pressure upon the median nerve. The histological evidence for repetitive hand activity provoking flexor tenosynovitis with any frequency in the community is not strong. ${ }^{9}$ It seems likely that most patients with CTS, whose work involves repetitive hand use have, at most, an exacerbation of an underlying constitutional tendency to nerve compression. An audit of patients requiring carpal tunnel decompression in Derby in 1989, revealed a higher frequency of CTS in non-working women of working age than in working women of working age ( 147 compared with 93 per 100000 of population per year), which suggests that work outside the home is not a major cause of CTS.

\section{TREATMENT OPTIONS \\ Wrist splints}

Wrist splints, used at night to hold the wrist in neutral flexion, are often of benefit in alleviating symptoms. The volume of the carpal tunnel varies with the position of the wrist and is maximal in neutral dorsipalmar flexion range. Crow advises that splintage may help up to $75 \%$ of cases and some patients can subsequently dispose of the splint without recurrent symptoms (this will tend to occur in mild cases with a short history of the condition). ${ }^{10}$

\section{Work modification}

Work modification, that avoids protracted periods of repetitive manual activities, can also be of benefit. Repetitive manual activities should be interspersed with alternative duties to provide a balance of activity.

\section{Non-steroidal anti-inflammatory agents}

Non-steroidal anti-inflammatory agents (NSAIDs) are not particularly effective in controlling symptoms for CTS. ${ }^{7}$

\section{Diuretics}

Diuretics have been advocated for many years. ${ }^{11}$ However, there is little evidence to support their use.

\section{Pyridoxine}

Pyridoxine (vitamin B12) has not been found to benefit the symptoms of CTS to any extent. ${ }^{12}$

\section{Steroid injections}

Steroid injections are commonly used in the diagnosis and the treatment of CTS. The procedure is hazardous and should only be performed by a doctor who is experienced with the anatomy of the carpal tunnel. Inadvertent steroid injection into the nerve is a serious error, which normally leads to chronic pain in the distribution of the median nerve. ${ }^{13}$ A steroid injection just proximal to the wrist crease is safer and equally effective. ${ }^{14} \mathrm{~A}$ point midway between the flexor carpi ulnaris and the palmaris longus is generally favoured. Several papers support a short term improvement in over $90 \%$ of cases. This makes it a useful diagnostic test if nerve conduction studies are equivocal or not readily available. However, only one quarter of patients show abiding benefit at one year and often the benefit does not exceed 4-6 months. ${ }^{15}$ A steroid injection can be particularly helpful in resolving CTS arising from pregnancy. The single injection will frequently resolve the symptoms in the last trimester, with continuing benefit postpartum. Steroid injections have the most prolonged effect in mild cases of CTS of recent onset. ${ }^{16}$ However, the benefit may be no greater than that obtained by splintage alone.

Kaplan and co-workers sought to identify predictors of poor outcome for conservative treatment. ${ }^{17} \mathrm{He}$ identified five factors that were associated with poor outcome: age over 50 years, symptoms in excess of 10 months, constant paraesthesia, triggering of digits, and a positive Phalen's test in 30 seconds or less. Medical treatment failed in $60 \%$ of cases if one of these factors was present, $83 \%$ if two factors were present, 93\% failed if three factors were present, rising to $100 \%$ if more than three factors were present.

Patients referred from their general practitioner with a provisional diagnosis of CTS are known to have a high "failure to attend" rate in the new patient clinics (M J Bradley, MSc thesis, University of Derby, 1999). Semistructured, in-depth, face to face interviews were carried out with 50 attenders and by telephone with 49 non-attenders. Contrary to expectation, those patients who had received a good explanation, and therefore reassurance, from the general practitioners concerning their diagnosis were more likely to fail to attend than those patients who had received little in the way of explanation. The non-attenders were able to make a rational decision, balancing the conflicts of interests between their concern for their hand problem and the other pressures in their lives regarding family, work, or other major health problems. This was in contrast to the patients who presented in the clinic who were still seeking further explanation and reassurance. Some general practitioners had spent time and had the knowledge of CTS to give good explanations to the patients. However, it would seem that they had not carried the consultation to its appropriate conclusion by really involving the patient in the decision to refer them to hospital.

Referrals would normally be on the basis of assessment for surgery, unless a steroid injection was considered to be worthwhile. Patients do need to be aware that they are being referred, most likely, for surgical intervention and they need to be in agreement with this concept. The authors therefore feel that any protocol for referral should include the proviso "refer if patient understands that surgery may be necessary" ( $\mathrm{J}$ Stothard, personal communication).

Surgical decompression of the carpal tunnel is the procedure of choice for all except the milder cases and medical treatment is not effective in controlling the moderate or 
severe cases. The outcome of surgical decompression is usually gratifying. A review of 186 cases revealed that $68 \%$ of patients were completely cured by surgical decompression, with $24 \%$ experiencing mild residual symptoms which the patient did not feel merited further treatment. ${ }^{18}$ The remaining $8 \%$ were left with recurrent or persistent problems of consequence and some were considered to have dual pathologies.

A protocol has been designed, on the basis of the available knowledge, with the aim of facilitating decision making in primary care. The protocol includes advice to primary care physicians in the form of 10 relevant clinical statements, which are based on the available relevant literature (box 1).

There is an additional statement emphasising the need to ensure that patients selected for referral truly understand the reasons and the need for referral and are a full partner in the referral process. The additional statement is included to minimise the risk of the patient failing to attend a new patient clinic.

The protocol can be applied in two ways, either through a decision tree (fig 1) or by making clinical choices on a referral template (fig 2).

The protocol is considered by the authors to be based on a reasonable assessment of the available literature. However, it is acknowledged that there are many areas where additional information is required. These include further studies on community prevalence, a measure of the spectrum of severity of symptoms and more extensive evidence of the efficacy of conservative treatment. It is also important to assess the impact that prolonged compression may have on outcome, after decompression.

\section{REFERENCES}

1 Burke FD, Dias JJ, Bradley M, et al. Providing care for hand disorders. The Derby Hand Unit experience 1989-90. J Hand Surg [Br] $1991 ; 16: 13-18$

2 Keller RB, Largay AM, Soule DN, et al. Maine carpal tunnel study-small area variations. J Hand Surg [Am] 1990;23:692-6.

3 Nordstrum DL, De Stefano F, Vierkant RA, et al. Incidence of diagnosed carpal tunnel syndrome in a general population. Epidemiology

4 De Krom MC, Knipschild PG, Kester AD, et al. Carpal tunnel syndrome prevalence in the general population. Br J Rheumatol 1998;37:630-5.

5 Ferry S. Pritchard T, Keenan J, et al. Estimating the prevalence of delayed median nerve conduction in the general population. BrJ Rheumatol 1998;37:630-5.

6 Burke FD. Carpal tunnel syndrome-reconciling "demand management" with clinical need. J Hand Surg [Br] 2000:25:121-7.

7 Rosenbaum RB, Ochoa JL. Carpal tunnel syndrome and other disorders of the median nerve. Boston: Butterworth Heinmann, 1993: 71-89.

8 Stromberg T, Dahlin LB, Brun A, et al. Structural nerve changes at wrist level in workers exposed to vibration. Occup Environ Med 1997;54:307-11.

9 Kerr CD, Sybert DR, Albarracin NS. An analysis of the flexor synovium in idiopathic carpal tunnel syndrome. J Hand Surg [Am] 1992; 17:1028-30.

10 Crow RS. Treatment of carpal tunnel syndrome. BM 1960;i:1611-15.

11 Guly PJ. Carpal tunnel syndrome. BMU 1959;i: 1184

12 Amadio PC. Pyridoxin as an adjunct to the treatment of carpal tunnel syndrome. J Hand Surg [Am] 1978;10:237-41.

13 Tavares SP, Giddins GEB. Nerve injury following steroid injection for carpal tunnel syndrome. J Hand Surg [Br] 1996;21:208-9.

14 Dammers JWHH, Veering MM, Vermenlen M. Injection of methyl Dammers JWH, Veering $M M$, Vermenlen $M$. Injection of methy
prednisolone proximal to the carpal tunnel: randomised double blind prednisolone proximal to the
trial. BM 1999:319:884-6.

15 Gelberman RH, Aronson D, Weisman NH. Carpal tunnel syndrome-results of a prospective trial of steroid injection and splintage. J Bone Joint Surg Am 1998;62:1 181-4

16 Babu SR, Britton JM. The role of steroid injection in the management of carpal tunnel syndrome. Journal of Orthopaedic Rheumatology 1994:7:59-60.

17 Kaplan SJ, Glickel SZ, Eaton RG. Predictive factors in the non-surgical treatment of carpal tunnel syndrome. J Hand Surg [Br] 1990;15:106-8.

18 Burke FD, Dias JJ, Webster H. Median nerve compression syndrome at the wrist. In: Hunter J, Schneider LH, Mackin EJ, eds. Tendon and nerve surgery in the hand. A third decade. St Lovis: Mosby, 1997: 145-51. 